Bundesgesundheitsbl 2020 $63: 1502-1510$ https://doi.org/10.1007/s00103-020-03240-6 Eingegangen: 18. Juni 2020

Angenommen: 12 . Oktober 2020

Online publiziert: 13 . November 2020

(c) Der/die Autor(en) 2020

Bianka Ditscheid' ' Markus Krause' ${ }^{1}$ Thomas Lehmann ${ }^{2}$ Kathleen Stichling' Maximiliane Jansky ${ }^{3}$. Friedemann Nauck $^{3}$. Ulrich Wedding ${ }^{4}$. Werner Schneider ${ }^{5}$.

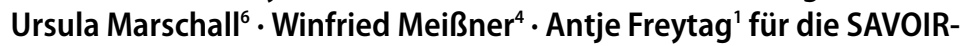
Studiengruppe

${ }^{1}$ Institut für Allgemeinmedizin, Universitätsklinikum Jena, Jena, Deutschland

${ }^{2}$ Zentrum für Klinische Studien, Universitätsklinikum Jena, Jena, Deutschland

${ }^{3}$ Klinik für Palliativmedizin, Universitätsmedizin Göttingen, Göttingen, Deutschland

${ }^{4}$ Abteilung Palliativmedizin der Klinik für Innere Medizin II, Universitätsklinikum Jena, Jena, Deutschland

${ }^{5}$ Zentrum für Interdisziplinäre Gesundheitsforschung, Universität Augsburg, Augsburg, Deutschland

${ }^{6}$ BARMER, Wuppertal, Deutschland

\title{
Palliativversorgung am Lebensende in Deutschland
}

\section{Inanspruchnahme und regionale Verteilung}

\section{Zusatzmaterial online}

Zusätzliche Informationen sind in der Online-Version dieses Artikels (https://doi. org/10.1007/s00103-020-03240-6) enthalten.

\section{Einführung}

Das Angebot an Palliativversorgung (PV) in Deutschland wurde in den letzten 20 Jahren stark ausgebaut. Besondere Aufmerksamkeit kam der spezialisierten ambulanten PV (SAPV) zu, die mit dem GKV(gesetzliche Krankenversicherung)-Wettbewerbsstärkungsgesetz 2007 Erstattungsfähigkeit durch die gesetzliche Krankenversicherung erlangte $(\$ 37 \mathrm{~b}$ SGB V). Dabei ergänzt sie „.. das bestehende Versorgungsangebot, insbesondere das der Vertragsärzte, Krankenhäuser und Pflegekräfte" ( $\$ 1$ Abs. 6 der SAPV-Richtlinie [1]). Gleichzeitig stieg die Anzahl an Hospizen und weitere Palliativstationen an Krankenhäusern wurden eingerichtet. Spezifische Qualifizierungsmaßnahmen für ärztliche und pflegerische Leistungserbringer wurden ausgeweitet und über alle Formen der PV hinweg wurden Leistungen besser vergütet. Dies gilt auch für die allgemeine ambulante PV (AAPV), die v. a. durch Hausärzte ${ }^{1}$, ergänzend auch durch niedergelassene Fachspezialisten, insbesondere Onkologen, getragen wird.

Die Berichterstattung zur Inanspruchnahme von PV am Lebensende wird derzeit im Wesentlichen durch öffentliche Statistiken getragen (SAPV-Frequenzstatistik, Kassenärztliche Bundesvereinigung; KG3-Statistik, DRG[Diagnosis Related Groups]-Statistik, gbe-bund.de). Hierbei fehlt jedoch der Personenbezug: Die SAPV-Frequenzstatistik zählt ausgestellte SAPV-Erst- und Folgeverordnungen personenunabhängig; SAPVVerordnungen aus dem Krankenhaus, die für bis zu 7 Tage ausgestellt werden können, werden nicht erfasst. Die KG3Statistik der SAPV-Abrechnungsfälle erfolgt quartalsweise; Fälle, die über Quartalsgrenzen verlaufen, werden in beiden Quartalen gezählt. Auch die DRG-Statistik ist fallbezogen. Die AAPV wurde bisher in keiner öffentlichen Statistik erfasst [2]. Neben der Berichterstattung zur PV für Deutschland insgesamt [3] wurden in einzelnen Bundesländern Gutachten zur regionalen Hospiz- und Palliativversorgung erstellt [4-8].

Auf Versichertenebene wurde die Inanspruchnahme von PV bisher nur aus

\footnotetext{
1 Für eine bessere Lesbarkeit wird nur eine sprachliche Form genutzt; hierbei sind Angehörige aller Geschlechter eingeschlossen.
}

der Kassenärztlichen Vereinigung (KV) Nordrhein berichtet [9]. In anderen KVRegionen und bundesweit wurde sie bislang nur von Radbruch et al. analysiert [10]. Die Grundlage bildeten dabei EBM-Ziffern (Einheitlicher Bewertungsmaßstab): Die SAPV-Inanspruchnahme wurde auf Basis der SAPV-Erst- und Folgeverordnungen, die AAPV-Inanspruchnahme auf Basis der Ziffern des EBM-Kapitels „Palliativmedizinische Versorgung" ermittelt.

Für AAPV-Leistungen und SAPVLeistungen gibt es neben EBM-Ziffern auch (selektivvertragliche oder KVspezifische) Sonderziffern, die für die Ermittlung der Inanspruchnahme von PV auf Versichertenebene ebenfalls zu berücksichtigen sind. Zudem können SAPV-Leistungen auch ohne eine in den Abrechnungsdaten dokumentierte Verordnung erbracht werden, z. B. wenn die Verordnung durch einen Krankenhausarzt ausgelöst wurde. Deshalb müssen auch SAPV-Leistungsabrechnungen herangezogen werden, um die Inanspruchnahme palliativer Leistungen möglichst vollständig darzustellen. Hospizleistungsabrechnungen können das Bild weiter vervollständigen.

Die Kenntnis über die tatsächliche Inanspruchnahme palliativer Versorgungsformen ist eine wichtige empirische Grundlage für die Weiterentwick- 
Tab. 1 Verteilung der Studienpopulation ${ }^{\text {a }}$ auf die palliativen Versorgungsformen in Abhängigkeit von der Bezugsgrundlage, alters- und geschlechtsstandardisiert

\begin{tabular}{|c|c|c|c|c|}
\hline \multirow[t]{2}{*}{ Bezugsgrundlage } & \multicolumn{4}{|c|}{ Anteil Versicherte in den Versorgungsformen [\%] } \\
\hline & AAPV & SAPV & Stationäre PV & Hospiz \\
\hline EBM-Ziffern für SAPV-VO sowie AAPV-Leistungen \& Krankenhaus-OPS-Codes & 19,3 & 7,1 & 8,1 & - \\
\hline $\begin{array}{l}\text { ZUSÄTZLICH regionale Sonderziffern sowie SAPV- und Hospizleistungsabrech- } \\
\text { nungen }\end{array}$ & 24,4 & 13,1 & 8,1 & 3,3 \\
\hline \multicolumn{5}{|c|}{$\begin{array}{l}\text { Quelle: eigene Berechnungen auf der Grundlage von BARMER-Daten } \\
\text { AAPV allgemeine ambulante Palliativversorgung, SAPV spezialisierte ambulante Palliativversorgung, PV Palliativversorgung, EBM Einheitlicher Bewertungs- } \\
\text { maßstab, OPS Operationen- und Prozedurenschlüssel } \\
{ }^{2} \text { Studienpopulation: im Jahr } 2016 \text { verstorbene Versicherte der BARMER, } N=95.962\end{array}$} \\
\hline
\end{tabular}

lung der Rahmenbedingungen für eine bedarfsgerechte und wirtschaftliche PV in allen Regionen Deutschlands. Ziel der vorliegenden Arbeit ist es, ein umfassenderes Bild der PV am Lebensende in Deutschland zu geben: Welche Art(en) der PV erhalten GKV-Versicherte am Lebensende und wie variiert dies regional?

\section{Methoden}

Als Teilprojekt des G-BA-innovationsfondsgeförderten Projekts SAVOIR [11], FKZ 01VSF16005, führten wir eine retrospektive Kohortenstudie mit GKV-Abrechnungsdaten durch, die im wissenschaftlichen Datawarehouse der BARMER bereitgestellt wurden. Primäres Einschlusskriterium für die Studienpopulation war ein Todesdatum im Jahr 2016.

Die Zuordnung der Versicherten zu den Kohorten erfolgte anhand einer mindestens einmaligen Abrechnung einer spezifischen Ziffer innerhalb der letzten 6 Lebensmonate: $A A P V$ wurde über die EBM-Ziffern für palliativmedizinische Versorgung (s. EBM, Kapitel 3.2.5.) und zusätzlich über KV-spezifische und selektivvertragliche Sonderziffern identifiziert. $S A P V$ identifizierten wir einerseits über EBM-Ziffern der SAPV-Erstund Folgeverordnung und andererseits über KV-spezifische und selektivvertragliche Sonderziffern sowie SAPVLeistungsabrechnungen. Stationäre Palliativversorgung wurde über die entsprechenden Codes für palliativmedizinische Prozeduren des Operationen- und Prozedurenschlüssels (OPS) identifiziert. Stationäre Hospizleistungen ( $\$ 39$ a Abs. 1
SGB V) wurden anhand von Hospizleistungsabrechnungen erfasst.

Eine detaillierte Beschreibung der Aufgriffskriterien findet sich im OnlineZusatzmaterial zu diesem Beitrag.

Die Daten wurden auf Bundesebene sowie auf Ebene der KV-Region der Versicherten deskriptiv mittels SAS Enterprise Guide Version 7.13 (SAS Institute Inc./Cary/NC/USA) ausgewertet. Alle Ergebnisse wurden standardisiert auf die Alters- und Geschlechtsstruktur aller im Jahr 2016 in Deutschland Verstorbenen (destatis.de).

\section{Ergebnisse}

\section{Studienpopulation}

Insgesamt wurden 95.962 Versicherte (=Verstorbene) eingeschlossen. Das entspricht einem Anteil von 10,7\% aller 2016 in Deutschland Verstorbenen ab 20 Jahre (destatis.de). Die Versicherten waren zum Todeszeitpunkt im Mittel $78,3 \pm 13,6$ Jahre alt; der Frauenanteil betrug 50,8 \%; 29,5\% waren Pflegeheimbewohner. (Zur Verteilung der Studienpopulation über die einzelnen $\mathrm{KV}$ Regionen im Vergleich zur Verteilung aller Verstorbenen auf die Bundesländer siehe Online-Zusatzmaterial, eTab. 1.).

\section{Inanspruchnahme von Palliativversorgung}

$32,7 \%$ der Versicherten erhielten in den letzten 6 Lebensmonaten mindestens eine palliative Leistung; ohne Sonderziffern und SAPV- sowie Hospizleistungsabrechnungen, d.h. unter ausschließlicher Berücksichtigung von EBM-Ziffern für AAPV-Leistungen und
SAPV-Verordnungen, betrug dieser Anteil $27,1 \%$. AAPV wurde bei $24,4 \%$ der Versicherten abgerechnet, wobei 5,1\% über Sonderziffern identifiziert wurden. $16,5 \%$ der Versicherten erhielten als ambulante PV ausschließlich AAPV (ohne SAPV). SAPV-Leistungen nahmen $13,1 \%$ der Versicherten in Anspruch, gut die Hälfte davon identifiziert anhand von SAPV-Erst- und Folgeverordnungen $(7,1 \%)$. 5,2\% der Versicherten erhielten nur SAPV (ohne AAPV). Unsere Analysen ergaben, dass bei $20,6 \%$ der Versicherten, die SAPV-Leistungen erhielten, die entsprechende Verordnung im Krankenhaus erfolgte. Bei 8,1\% der Versicherten wurden stationäre palliativmedizinische Leistungen abgerechnet; 3,0\% erhielten ausschließlich stationäre palliativmedizinische Leistungen. Stationäre Hospizleistungen wurden von 3,3\% der Versicherten in Anspruch genommen (• Tab. 1).

\section{Inanspruchnahme nach KV- Regionen}

Die Auswertung der Inanspruchnahme von PV auf der Ebene der KV-Region der Versicherten zeigte deutliche regionale Unterschiede (• Abb. 1 und $2 \mathrm{a}-\mathbf{d}$ ). In Bayern und in Niedersachsen erhielt im Vergleich zum Bundesdurchschnitt ein hoher Anteil an Versicherten am Lebensende PV, während in Bremen, SachsenAnhalt und Sachsen anteilig deutlich weniger Versicherte palliativ versorgt wurden (• Abb. 1).

Der Anteil an Versicherten mit $A A P V$ war in Bayern mit 34,1\% um fast 10 Prozentpunkte höher als im Bundesdurchschnitt. Auch in Niedersachsen erhielt mit 28,1\% ein vergleichsweise hoher An- 
Bundesgesundheitsbl 2020 · 63:1502-1510 https://doi.org/10.1007/s00103-020-03240-6

(c) Der/die Autor(en) 2020

B. Ditscheid · M. Krause · T. Lehmann · K. Stichling · M. Jansky · F. Nauck · U. Wedding · W. Schneider · U. Marschall · W. Meißner · A. Freytag für die SAVOIR-Studiengruppe

\section{Palliativversorgung am Lebensende in Deutschland. Inanspruchnahme und regionale Verteilung}

Zusammenfassung

Hintergrund. Das Angebot an Palliativversorgung hat in Deutschland stark zugenommen. Weitgehend unbekannt ist, wie viele Menschen am Lebensende welche palliativen Versorgungsformen in Anspruch nehmen und welche regionalen Unterschiede bestehen. Methode. Retrospektive Kohortenstudie mit GKV-Routinedaten (BARMER) über Versicherte mit Sterbedatum im Jahr 2016: Anhand einer mindestens einmalig abgerechneten Leistung in den letzten 6 Lebensmonaten wurde die Inanspruchnahme allgemeiner ambulanter Palliativversorgung (AAPV), spezialisierter ambulanter Palliativversorgung (SAPV) sowie stationärer Palliativ- und Hospizversorgung ermittelt. Erstmals wurden auch Abrechnungsziffern kassenärztlicher Vereinigungen und selektivvertragliche Sonderziffern für palliativmedizinische Leistungen sowie SAPVLeistungsabrechnungen herangezogen. Ergebnisse. Von den 95.962 Verstorbenen der Studienpopulation wurden bundesdurchschnittlich $32,7 \%$ palliativ versorgt, mit Schwankungen zwischen $26,4 \%$ in Bremen und $40,8 \%$ in Bayern. AAPVLeistungen wurden bei $24,4 \%$ abgerechnet $(16,9 \%$ in Brandenburg bis $34,1 \%$ in Bayern). SAPV-Leistungen erhielten 13,1\% $(6,3 \%$ in Rheinland-Pfalz bis $18,9 \%$ in Brandenburg bzw. 22,9\% in Westfalen-Lippe mit abweichender SAPV-Praxis). Stationär palliativmedizinisch versorgt wurden $8,1 \%$ (6,7\% in Schleswig-Holstein/Hessen bis 13,0\% in Thüringen), Hospizleistungen wurden für
3,3 \% abgerechnet (1,6\% in Bremen bis 5,6\% in Berlin).

Diskussion. SAPV wird häufiger in Anspruch genommen als bisher berichtet, AAPV ist rückläufig. Die jeweilige Inanspruchnahme scheint weniger durch objektiven Bedarf als durch regionalspezifische Rahmenbedingungen begründet. Die Weiterentwicklung der Palliativversorgung sollte zukünftig neben Bedarfskriterien mehr an Outcomes sowie dafür relevanten Rahmenbedingungen orientiert werden.

Schlüsselwörter

Ambulante Palliativversorgung · AAPV · SAPV . GKV-Routinedaten · Regionale Unterschiede

\section{Palliative care at the end of life in Germany. Utilization and regional distribution}

\section{Abstract}

Background. Palliative care supply increased in Germany in recent years. But how many people use which forms of palliative care and how does this differ between regions? Method. Retrospective cohort study with claims data from insured persons who died in 2016: Based on services billed at least once in the last six months of life, we determined the use of primary palliative care (PPC), specialized palliative homecare (SPHC), as well as inpatient palliative and hospice care, using regional billing codes for PPC and SPHC services for the first time.
Results. Of the 95,962 deceased in the study population, $32.7 \%$ received palliative care nationwide, with variations from $26.4 \%$ in Bremen to $40.8 \%$ in Bavaria. PPC services were billed at $24.4 \%$ (16.9\% in Brandenburg to $34.1 \%$ in Bavaria). SPHC services received 13.1\% (6.3\% in Rhineland-Palatinate to $18.9 \%$ in Brandenburg and $22.9 \%$ in WestphaliaLippe with different SPHC practices). Inpatient palliative care was received by $8.1 \%$ (6.7\% in Schleswig-Holstein/Hesse to $13.0 \%$ in Thuringia); $3.3 \%$ (1.6\% in Bremen to $5.6 \%$ in Berlin) with hospice services.
Conclusion. SPHC is used more frequently than previously reported, while PPC is declining. Utilization seems to be based less on objective needs than on region-specific framework conditions. Besides needs criteria, further development of palliative care should be oriented more towards outcomes and relevant framework conditions.

Keywords

Palliative homecare · PPC · SPHC · Claims data . Regional comparison teil an Versicherten vor dem Tod AAPV. Im Gegensatz dazu lag der Anteil an Versicherten mit AAPV-Leistungen in Brandenburg (16,9\%), Sachsen-Anhalt $(17,0 \%)$, Sachsen $(18,0 \%)$ und Berlin (18,6\%) deutlich unter dem Bundesdurchschnitt (- Abb. 2a).

Für $S A P V$-Leistungen zeigten sich noch deutlich stärkere Unterschiede zwischen den KV-Regionen. Mit nur 6,3\% war der Anteil an Versicherten mit SAPV in Rheinland-Pfalz weniger als halb so hoch wie im Bundesdurchschnitt. Auch in Bremen (7,4\%), Nordrhein (9,0\%), Baden-Württemberg $(9,7 \%)$ und Bayern $(10,0 \%)$ erhielten deutlich weniger Ver- sicherte SAPV-Leistungen als im Bundesdurchschnitt. Einen hohen Anteil an Versicherten mit SAPV-Leistungen wiesen hingegen Brandenburg (18,9\%), Berlin (16,7\%) und Hessen (16,4\%) auf. Die Leistungen des Palliativmedizinischen Konsiliardienstes (PKD) in Westfalen-Lippe wurden von 22,9\% der Versicherten in Anspruch genommen (- Abb. 2b).

Stationäre $P V$ im Krankenhaus wurde von Versicherten der KV-Regionen Thüringen (13,0\%), Mecklenburg-Vorpommern und Saarland (je 11,3\%) sowie Brandenburg (10,6\%) anteilig häufiger und seltener in Schleswig-Holstein,
Hessen (je 6,7\%) und Nordrhein (6,8\%) in Anspruch genommen (-Abb. 2c).

Stationäre Hospizleistungen wurden in Bremen (1,6\%), Bayern (2,0\%) und Schleswig-Holstein $(2,1 \%)$ anteilig weniger in Anspruch genommen, während der Anteil in Berlin (5,6\%), Hamburg $(5,4 \%)$ und Mecklenburg-Vorpommern $(5,0 \%)$ über dem Bundesdurchschnitt lag (• Abb. 2d).

\section{Diskussion}

Unsere Analyse liefert ein nach KVRegionen differenziertes Bild der Palliativversorgung (PV) in Deutschland. 


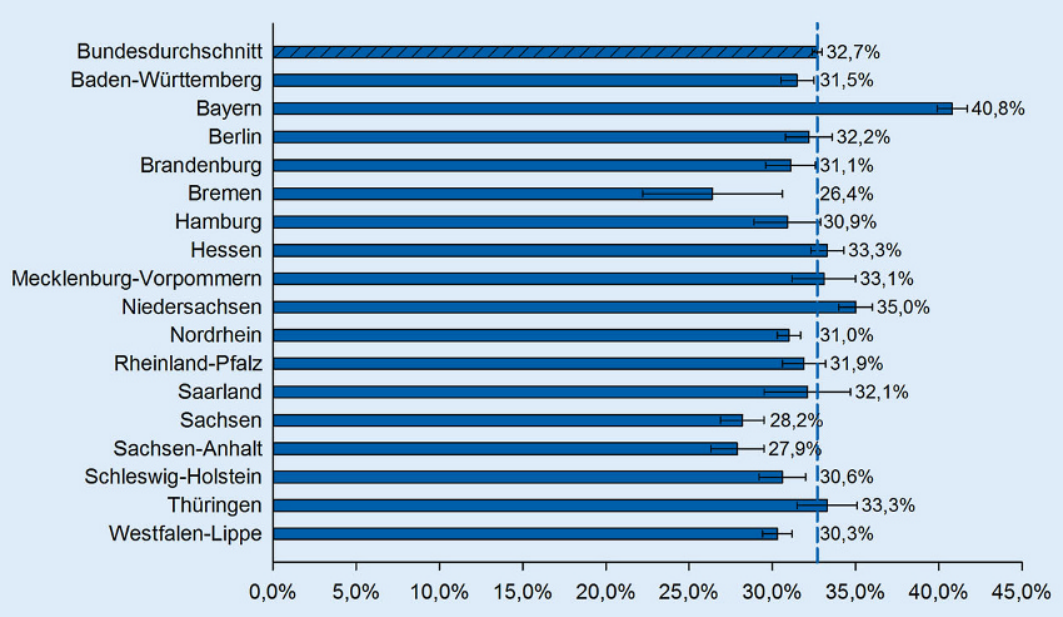

Abb. $1 \Delta$ Anteil Versicherte mit Palliativversorgung in den letzten 6 Lebensmonaten, 2016, stratifiziert nach KV-Region, alters- und geschlechtsstandardisiert (inkl. $95 \%$-Konfidenzintervall; vertikale, gestrichelte Linie: Bundesdurchschnitt). Quelle: eigene Berechnungen auf der Grundlage von BARMERDaten

Die Inanspruchnahme verschiedener Formen der PV am Lebensende auf Versichertenebene wurde anhand von Krankenkassenabrechnungsdaten quantifiziert. Dabei wurden erstmals auch Sonderziffern und SAPV- sowie Hospizleistungsabrechnungen herangezogen. Die Ergebnisse zeigen, dass deren Berücksichtigung essenziell ist: Der Anteil an Versicherten mit SAPV-Leistungen betrug im Jahr 2016 nicht 7,1\% wie auf Basis der Verordnungen ermittelt, sondern war mit $13,1 \%$ beinahe doppelt so hoch. Und auch dieser Anteil dürfte noch unterschätzt sein, da SAPVAbrechnungen für die KV Berlin nicht vollständig zur Verfügung standen. Bei etwa einem Fünftel der Versicherten mit SAPV wurde die SAPV im Krankenhaus verordnet - ein großer Anteil, der bislang nicht quantifiziert wurde.

Der Anteil der Versicherten, der in den letzten 6 Lebensmonaten AAPV erhielt, wurde erstmals umfassend geschätzt und beträgt $24,4 \%$. Er ist aber wegen vermuteter Lücken bei der Identifikation von Vergütungsziffern integrierter Versorgungsverträge sowie einer möglicherweise lückenhaften Dokumentation palliativmedizinischer Leistungen durch Hausärzte ebenfalls eher unterschätzt. Bei der Versorgung älterer, gebrechlicher Patienten sieht das Vergütungssystem nämlich eine Trennung zwischen geriatrischen und palliativmedizinischen
Leistungen vor, die in der hausärztlichen Praxis verschmelzen [12], was mit einer Unterdokumentation palliativmedizinischer Leistungen einhergehen kann [13].

\section{Vergleich mit Bedarfsschätzungen und im zeitlichen Verlauf}

Der Anteil von 32,7\% der Versicherten mit mindestens einer Palliativleistung in den letzten 6 Lebensmonaten liegt noch immer deutlich unter dem geschätzten Bedarf für Deutschland: Unter Verwendung der Schätzmethode von Murtagh et al. [14] wiesen $78 \%$ der Todesfälle im Jahr 2013 einen palliativen Versorgungsbedarf auf [15]. Auf internationaler Ebene wird der Bedarf auf 56-83\% geschätzt [16].

Laut Schätzungen der Deutschen Gesellschaft für Palliativmedizin (DGP) haben sogar $90 \%$ der Versterbenden palliativen Versorgungsbedarf, davon wiederum etwa $10 \%$ Bedarf an spezialisierter PV $[10,17]$. Diesem geschätzten Bedarf von $90 \%$ bzw. $10 \%$ steht in unseren Analysen eine Inanspruchnahme von 32,7\% bzw. 13,1 \% gegenüber. Demnach bestünde insgesamt eine deutliche palliative Unterversorgung bei einer gleichzeitig über der genannten Bedarfsschätzung liegenden Inanspruchnahme von SAPV.

Mit 13,1\% erhielten im Jahr 2016 deutlich mehr Menschen vor ihrem Tod
SAPV-Leistungen als bisher berichtet (im Mittel 3,5\% für die Jahre 2010-2014 [10]) bzw. als es auf Basis der SAPVVerordnungen ermittelbar war (7,1\%). Dies deckt sich mit dem Anstieg der Anzahl an SAPV-Verträgen bundesweit von 261 auf $293(+12,3 \%)$ zwischen 2014 und 2016 [18]. Die starke Verbreitung der SAPV zeigt sich schließlich auch in der Gegenüberstellung der SAPVInanspruchnahme von $13,1 \%$ mit der alleinigen Inanspruchnahme von AAPV von nur $16,5 \%$.

Der Anteil von Versicherten mit AAPV-Leistungen lag mit 24,4\% im Jahr 2016 auf demselben Niveau wie es von Radbruch et al. [10] für 2014 berichtet wurde. Zieht man aber - wie ebendort - ausschließlich die EBMZiffern der AAPV heran, so lag die von uns ermittelte Inanspruchnahme im Jahr 2016 bei 19,3\%. Während also die Inanspruchnahme von SAPV stark zugenommen hat, ist bei AAPV ein Rückgang zu verzeichnen, und dies obwohl der geschätzte Bedarf an PV insgesamt bei Weitem nicht gedeckt ist.

\section{Regionaler Vergleich}

Erkennbar ist eine starke regionale $\mathrm{He}$ terogenität in der Inanspruchnahme unterschiedlicher Formen der PV, hier insbesondere im Verhältnis allgemeiner zu spezialisierter Versorgung.

Potenzielle Einflussfaktoren stellen neben Bedarfsunterschieden und nachfrageseitigen Unterschieden z.B. in der wahrgenommenen Qualität oder Kenntnis der Angebote (bei Patienten/Angehörigen wie auch betreuenden Versorgern) insbesondere regionsspezifische Rahmenbedingungen wie Versorgungskulturen und Leistungs- und Vergütungsbedingungen palliativer Versorgungsformen dar, aus denen regional unterschiedliche Angebotsstrukturen hervorgehen. Für die SAPV gilt dabei eine nur eingeschränkte interregionale Vergleichbarkeit in Leistungsart und -umfang [17] aufgrund unterschiedlicher Vertragsbedingungen [19]. In geringerem Maße gelten solche Unterschiede auch für die AAPV, wenn KVoder selektivvertragliche Sonderziffern existieren. Der grundsätzlich höhere 


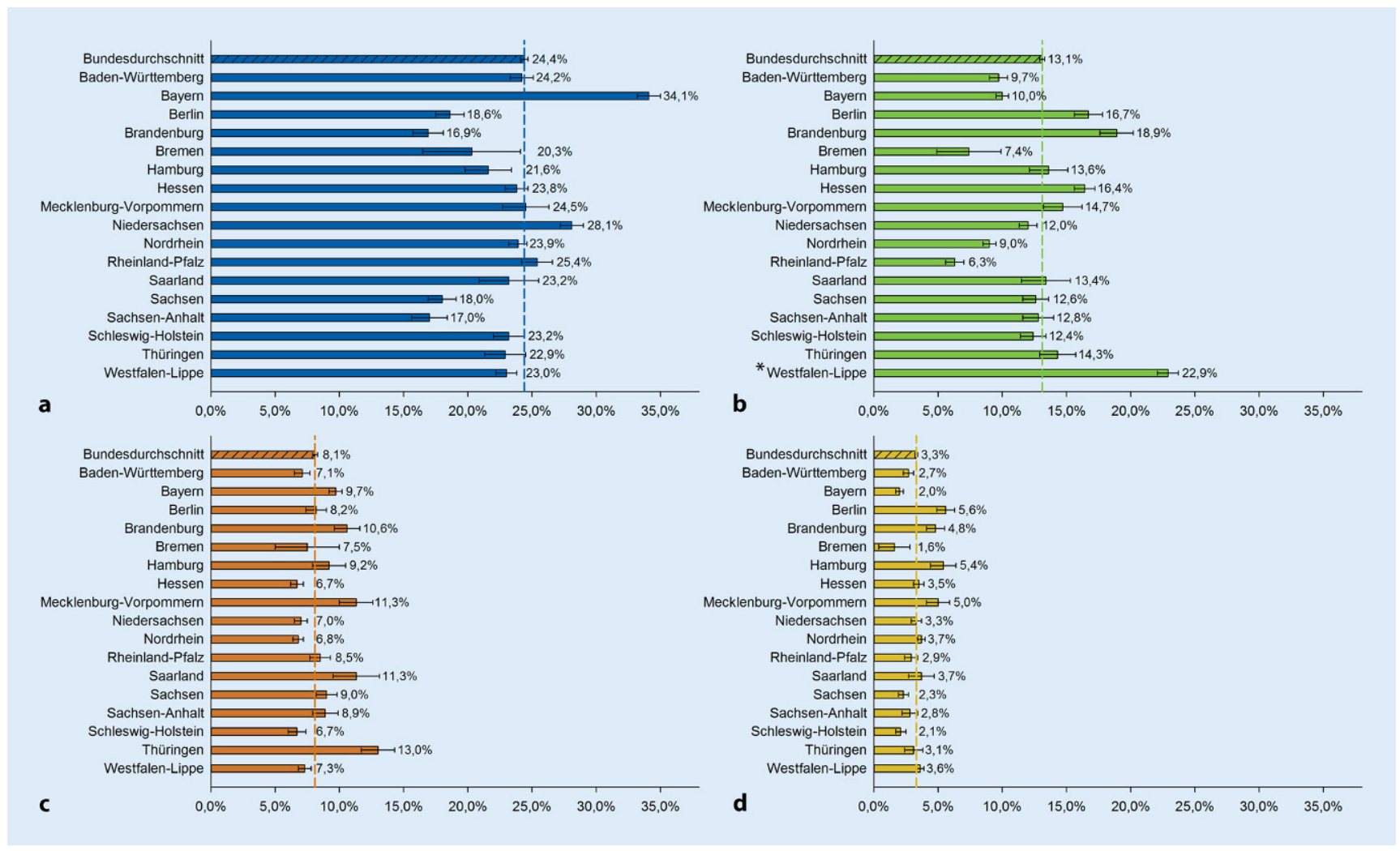

Abb. $2 \Delta$ Anteil Versicherte mit Inanspruchnahme verschiedenerFormen derPalliativversorgung (PV) in den letzten 6Lebensmonaten, 2016, stratifiziert nach KV-Regionen, alters- und geschlechtsstandardisiert (inkl. $95 \%$ Konfidenzintervall; vertikale, gestrichelte Linie: Bundesdurchschnitt): a Allgemeine ambulante PV (AAPV), b spezialisierte ambulante PV (SAPV), c stationäre PV, d stationäre Hospizleistungen. Quelle: eigene Berechnungen auf der Grundlage von BARMER-Daten. ( $*$ Leistungen des palliativmedizinischen Konsiliardienstes (PKD) werden hier als SAPV-Leistungen erfasst, siehe Ausführungen dazu im OnlineZusatzmaterial)

Spezialisierungs- und Anforderungsgrad ebenso wie eine höhere Vergütung von SAPV-Leistungen gegenüber AAPVLeistungen ist jedoch durchgängig in allen Regionen vorhanden. Während für jedes Hospiz ein eigener Vergütungssatz (tagesbezogener Bedarfssatz) mit den Kassenverbänden auf Landesebene vereinbart wird [20], werden Palliativleistungen im Krankenhaus überwiegend bundeseinheitlich vergütet (bis auf unterschiedliche Landesbasisfallwerte und bundeslandspezifische Zusatzentgelte für besondere Einrichtungen).

Der Zusammenhang zwischen Inanspruchnahme und Angebotskapazitäten zeigt sich eindrucksvoll in der nahezu exakten Deckung regionaler Inanspruchnahme stationärer PV im Krankenhaus (s. Ergebnisse) mit dem jeweiligen Angebot an Palliativbetten [21]. Beinahe ebenso deutlich deckt sich das Angebot an Hospizbetten [21] mit der Inan- spruchnahme stationärer Hospizleistungen (s. Ergebnisse).

Für die jeweilige Entscheidung, ob AAPV oder SAPV zum Einsatz kommt, liegen Hinweise vor, dass diese in hohem Maße von den verfügbaren hausärztlichen AAPV-Kapazitäten abhängt [22]. Weitere Faktoren zur Erklärung regionalspezifischer Inanspruchnahmemuster sollen im Folgenden für ausgewählte KV-Regionen mit niedriger bzw. hoher Inanspruchnahme von AAPV bzw. SAPV erörtert werden:

Bayern fällt durch die mit $34,1 \%$ im Bundesvergleich sehr hohe Inanspruchnahme von AAPV auf. Hier gab es bereits sehr früh eine starke ambulante Hospizkultur. Die damit einhergehenden Netzwerke (der Hospiz- und Palliativversorgung) haben die regionale palliative Versorgungslandschaft geprägt [17]. 2011 wurde das „Rahmenkonzept zur Hospiz- und Palliativversorgung" veröffentlicht [23]. Der Grundsatz „am- bulant vor stationär" sowie die multiprofessionelle Vernetzung auch über Sektorengrenzen hinweg spielen eine wesentliche Rolle. Hinzu kommt, dass die hausarztzentrierte Versorgung ( $\$ 73 \mathrm{~b}$ SGB V) in Bayern gut etabliert ist und eigene Vergütungsziffern für AAPVLeistungen vorsieht. Im Vergleich dazu lag die Inanspruchnahme von SAPV unter dem Bundesdurchschnitt. Die SAPV-Teams sind zum Großteil auf Initiative der o.g. Netzwerke bzw. der Hospiz- und Palliativvereine entstanden und fügen sich damit in die vorhandene Netzwerkstruktur ein. Im Vergleich zum Bundesdurchschnitt verfügt Bayern über deutlich weniger Ärzte mit Zusatzweiterbildung „Palliativmedizin“ (OnlineZusatzmaterial, eTab. 2). Dies ist womöglich auf spezifische Anforderungen in der Weiterbildungsordnung der Bayerischen Landesärztekammer für diese Zusatzweiterbildung zurückzuführen. 
Die Mehrheit der Palliativmediziner ist stationär tätig (eTab. 2).

Berlin fällt durch die mit $16,7 \%$ hohe Inanspruchnahme von SAPV auf. Bereits seit 1992 wurden hier Vereinbarungen nach $₫ 73$ a SGB V für die Präfinalversorgung von Krebs- oder Aidspatienten geschlossen - die sog. Home-Care-Versorgung [24]. Die SAPV baute auf diesen Strukturen auf [24]: Home Care Berlin e. V. (=Berliner Landesverband der Bundesarbeitsgemeinschaft SAPV) koordiniert die in der SAPV tätigen Ärzte, Pflegedienste sowie weitere Leistungserbringer, die in offenen Teams zusammenarbeiten. Dabei dürfen die spezialisierten Palliativärzte SAPV-Folgeverordnungen selbst ausstellen und abrechnen (s. Rahmenvertrag für Berlin aus dem Jahr 2013, $\$ 6$ Abs. 4), ggf. auch für Aufgaben, die in anderen Bundesländern der AAPV zugerechnet werden [10]. Dies erklärt die hohe Inanspruchnahme von SAPV in Berlin. Demgegenüber war die Inanspruchnahme von AAPV-Leistungen hier mit $18,6 \%$ auffällig gering. Hausärzte sind deutlich weniger als in anderen Bundesländern in die PV involviert [25]. Oft läuft diese regelrecht an ihnen vorbei: Sobald erstmals SAPV verordnet wurde, werden sie nicht einmal mehr als Verordner benötigt. Verstärkt wird dieser Effekt noch dadurch, dass SAPV-Erstverordnungen auch häufig aus dem Krankenhaus heraus erfolgen. Berlin verfügt darüber hinaus über ein im Vergleich zu anderen Regionen höheres Angebot an Hospizbetten [21], das auch in Anspruch genommen wurde.

Brandenburg fällt durch die mit 16,9\% im Bundeslandvergleich geringste Inanspruchnahme von AAPV auf, auch wenn diese auf Landkreisebene in Abhängigkeit von lokalen Angeboten an Hospiz- und Palliativversorgung variiert [26]. Brandenburg hat zum einen im Bundeslandvergleich eine geringe Anzahl an Hausärzten (eTab. 2, [27]) und gleichzeitig eine im Vergleich zum Bundesdurchschnitt deutlich geringere Einwohnerdichte (eTab. 2). So entstehen je Arzt große Versorgungsradien. Der Anteil an Ärzten mit Zusatzweiterbildung „Palliativmedizin“ ist in Brandenburg vergleichsweise hoch (eTab. 2). Diese sind wegen der größeren Versorgungs- radien und besseren Vergütung wahrscheinlich eher im Rahmen der SAPV tätig. In Brandenburg erhielt mit 18,9\% ein überdurchschnittlich hoher Anteil an Versicherten SAPV-Leistungen. Fehlende AAPV-Strukturen werden daher vermutlich durch SAPV substituiert: Anstelle einer AAPV, die als solche ausreichend sein könnte, kommen SAPVTeams zum Einsatz, weil ansonsten gar keine ambulante PV möglich wäre.

In Hessen lag die Inanspruchnahme von SAPV mit 16,4\% deutlich über dem Bundesdurchschnitt, während die Inanspruchnahmevon AAPV mit 23,8\% in etwa dem Bundesdurchschnitt entsprach. Die SAPV hat sich in Hessen aus den seit 2005 vereinzelt abgeschlossenen Verträgen zur integrierten Versorgung Palliativmedizin entwickelt [28, 29]. SAPVTeams (Palliative Care Teams) sind zusammen mit Hausärzten, Pflegediensten, Hospizdiensten und Kliniken in Netzwerken organisiert. Hier liegt somit traditionell ein besonderes Augenmerk auf der Entwicklung adäquater integrierter PV-Strukturen, um den Versorgungsbedarf von Patienten zu decken, der die Leistungsfähigkeit der bestehenden Regelversorgung übersteigt, ohne bereits die Komplexität einer SAPV-Versorgung zu begründen [28, 29]. Im Bundesvergleich gehört Hessen zu den Bundesländern mit den meisten ambulant tätigen Palliativmedizinern (eTab. 2); die SAPV ist flächendeckend ausgebaut. Stationäre PV im Krankenhaus wurde hier eher weniger in Anspruch genommen.

Niedersachsen fällt durch den mit 28,1\% im Bundeslandvergleich hohen Anteil an Versicherten mit AAPV-Leistungen auf. Seit 2006 sind hier flächendeckend Palliativstützpunkte als Netzwerke der örtlichen Hospizarbeit und PV entstanden, innerhalb derer vorhandene Angebotsstrukturen kooperativ zusammenarbeiten [30, 31]. Die Palliativstützpunkte bündelten die Strukturen der Spezialversorgung, koordinierten deren Angebot und berieten die Leistungserbringer der Basisversorgung [30]. Damit gab es hier bereits gut vernetzte lokale Strukturen, bevor die SAPV bundesweit durch die GKV erstattungsfähig wurde. Die so gewachsenen Palliativstrukturen haben eine breite Verankerung der PV im Bereich der ambulanten ärztlichen Versorgung insgesamt mit sich gebracht. Niedersachsen verfügt im Bundeslandvergleich über die meisten ambulant tätigen Ärzte mit Zusatzweiterbildung „Palliativmedizin“. Auch ist deren Anteil an allen Ärzten mit ambulanter Tätigkeit hier am höchsten (eTab. 2). Lokale Ärztenetze (nach $₫ 87 \mathrm{~b}$ SGB V) fördern die Qualifizierung ihrer Ärzte zu Palliativmedizinern, bauen SAPV-Teams auf und gründen Palliativstützpunkte. Radbruch et al. sehen den vergleichsweise hohen Anteil an SAPV in Niedersachsen auch darin begründet, dass durch weniger strikte Struktur- und Prozessvorgaben in SAPV-Verträgen mehr Einrichtungen Leistungen der SAPV anbieten können [10]. In unseren Analysen lag die Inanspruchnahme von SAPV in Niedersachsen mit 12,0\% etwas unter dem Bundesdurchschnitt. Stationäre PV im Krankenhaus wurde in Niedersachsen vergleichsweise weniger in Anspruch genommen.

In Nordrhein entsprach die Inanspruchnahme von AAPV (s. auch Online-Zusatzmaterial) mit $23,9 \%$ in etwa dem Bundesdurchschnitt, während die von SAPV mit 9,0\% deutlich darunter lag. Hier wurde bereits frühzeitig auf eine flächendeckende Umsetzung der ambulanten palliativmedizinischen und -pflegerischen Versorgung im Sinne eines kooperativen integrativen Versorgungskonzepts gesetzt [32]. Dabei bilden Ärzte mit palliativmedizinischer Basisqualifikation (Absolvierung einer 40stündigen Kursweiterbildung Palliativmedizin) gemeinsam mit einem ambulanten palliativpflegerischen Dienst die Basis der Versorgung; bei Bedarf kann ein qualifizierter Palliativarzt (QPA, Voraussetzung: Zusatzbezeichnung „Palliativmedizin“) oder ein weiterer Kooperationspartner (z.B. Hospizdienst) hinzugezogen werden [32]. Mit Einführung des gesetzlichen Anspruchs auf SAPV entstanden sogenannte Palliative Care Teams als SAPV-Dienstleister, in denen QPÄ mit ausgebildeten Palliativpflegekräften zusammenarbeiten. Damit ist zwischen der im Rahmen der AAPV anforderbaren Einzelleistung eines QPA [33] und der Teamleistung der SAPV zu differenzieren [34]. Dies erklärt auch die 
geringere Inanspruchnahme von SAPV: Große Versorgungsanteile entfallen auf die vorgelagerte Spezialisierungsebene des QPA und damit der AAPV. An der Vereinbarung teilnehmende Haus- und Fachärzte mit palliativmedizinischer Basisqualifikation können als koordinierender Arzt fungieren und ebenfalls AAPV-Leistungen abrechnen [33]. Seit Aufnahme von Abrechnungsziffern für die hausärztliche PV in den EBM im 4. Quartal 2013 existiert in Nordrhein innerhalb der AAPV somit ein dreistufiges System aus Basisversorgung (EBM-Ziffern der Palliativversorgung), koordinierenden Ärzten mit palliativmedizinischer Basisqualifikation sowie QPÄ. Stationäre PV im Krankenhaus wurde in Nordrhein eher weniger in Anspruch genommen.

In Westfalen-Lippe wurden schon sehr früh und regelhaft spezielle ambulante Palliativstrukturen gefördert, die palliativmedizinischen Konsiliardienste (PKD). Diese wurden später an die bundesweite Einführung der SAPV angepasst. Die „Vereinbarung zur Umsetzung der ambulanten palliativmedizinischen Versorgung von unheilbar erkrankten Patienten im häuslichen Umfeld" [35] ermöglicht explizit Übergänge zwischen allgemeiner und spezialisierter Versorgung. Der Hausarzt bleibt hier auch in der palliativen Versorgungssituation der zentrale Ansprechpartner [36, 37]. Eingeschriebene Haus- und Fachärzte arbeiten direkt mit dem PKD zusammen, der die spezialisierte palliativmedizinische Versorgung bei Bedarf übernimmt. Eingeschriebene Ärzte können die AAPVZiffern dieser Vereinbarung abrechnen, nicht eingeschriebene Ärzte können nur die Ziffern der Regelversorgung (EBM-Ziffern) abrechnen. Das Hinzuziehen des PKD bedarf keiner SAPVVerordnung nach Muster 63 [35]. Die spezialisierten palliativmedizinischen Leistungen des PKD werden in dieser Studie der SAPV zugeordnet (siehe Online-Zusatzmaterial „eMethoden“). In unseren Analysen war Westfalen-Lippe mit 22,9\% diejenige KV-Region mit der höchsten Inanspruchnahme von SAPV (im Sinne einer Inanspruchnahme von PKD-Leistungen, s. o.g. Vereinbarung). Gleichzeitig erhielten aber auch 23,0\% der Versicherten am Lebensende AAPVLeistungen. Ausschließlich AAPV-Leistungen (ohne SAPV) erhielt lediglich ein Fünftel aller AAPV-Versorgten. Die Inanspruchnahme stationärer $\mathrm{PV}$ im Krankenhaus lag hingegen mit 7,3\% deutlich unter dem Bundesdurchschnitt.

Im Ergebnis lässt sich eine Gruppe an KV-Regionen identifizieren, in denen eine geübte, historisch gewachsene Zusammenarbeit der an der ambulanten PV mitwirkenden Akteure (Niedersachsen, Hessen) bzw. eine starke Verzahnung von allgemeiner und spezialisierter ambulanter Versorgung (Westfalen Lippe, Nordrhein) existiert. In diesen KV-Regionen ist die Inanspruchnahme stationärer PV eher gering. Dies könnte so gedeutet werden, dass starke, gewachsene, später um SAPV ergänzte ambulante Strukturen den palliativen Versorgungsbedarf zu decken vermögen, ohne vermehrt auf stationäre Kapazitäten zurückgreifen zu müssen. Anders gelagert ist der Fall Bayern, wo die gewachsenen allgemeinen ambulanten Strukturen in Kombination mit einer eher geringfügig ausgebauten SAPV die Inanspruchnahme stationärer Strukturen nicht verhindern.

In einer weiteren Gruppe an KV-Regionen überwiegt die SAPV gegenüber der AAPV, entweder weil AAPV-Versorgungslücken bestehen, d.h. nicht genügend Erbringer von AAPV vorhanden sind (Brandenburg), oder weil die spezialisierten Strukturen besonders leicht zugänglich sind und einen Einsatz der Hausärzte in AAPV überflüssig machen (Berlin).

\section{Limitationen}

Die Limitationen dieser Studie sind im Online-Zusatzmaterial zusammengefasst.

\section{Fazit}

Indem Leistungsziffern so umfassend wie möglich einbezogen wurden, stellen wir die Berichterstattung zur Inanspruchnahme der PV auf eine neue Grundlage: Mit 13,1\% erhielten deutlich mehr Menschen vor ihrem Tod SAPV-Leistungen als bisher berichtet und als bisherige Be- darfsschätzungen beziffern. Gleichzeitig ist die Abrechnung von AAPV-Leistungen stagnierend bzw. rückläufig. Für die stationäre PV ist festzustellen, dass vorhandene Palliativ- (und Hospiz-)Betten auch genutzt werden. Funktionierende ambulante Palliativstrukturen scheinen weniger stationäre Inanspruchnahme zu erfordern. Der insgesamt möglicherweise eher gering zu bewertende Anteil von Versicherten, die irgendeine Form von PV erhalten $(32,7 \%)$, erscheint am ehesten erweiterbar durch eine Stärkung der Strukturen allgemeiner PV.

Des Weiteren wird die starke regionale Heterogenität in der Inanspruchnahme der unterschiedlichen PV-Formen deutlich. Diese ist durch Bedarfsunterschiede allein kaum erklärbar. Vielmehr scheinen regionalspezifische Rahmenbedingungen wie historisch herausgebildete Versorgungskulturen sowie Leistungs- und Vergütungsbedingungen jeweils regionale Angebotsstrukturen hervorgebracht zu haben, die dann auch in Anspruch genommen werden. Regional unterschiedliche Strukturen sind nicht per se negativ. Bewertet werden sollten diese anhand ihrer Outcomes. Diese zu definieren, zu erfassen und zu vergleichen, ist Aufgabe zukünftiger Versorgungsforschung. Gegebenenfalls lassen sich dabei Best-Practice-Regionen identifizieren, deren Wirkelemente sich auch in anderen Regionen implementieren lassen.

Unsere anhand von verfügbaren Regionaldaten angestellte Untersuchung zur Erklärung der regionalen Inanspruchnahme palliativer Versorgungsformen sollte zukünftig anhand von schließenden statistischen Analysen überprüft werden, in denen (geschätzte) Bedarfe sowie weitere nachfrageund angebotsseitige Einflussfaktoren als unabhängige Größen Berücksichtigung finden. Dennoch lässt sich bereits jetzt schlussfolgern: Die Inanspruchnahme palliativer Versorgungsformen, vor allem aber eine Aufnahme in die allgemeine bzw. spezialisierte Versorgung, ist nicht allein durch medizinischen Bedarf erklärt. Deshalb sollte die Weiterentwicklung der PV zukünftig nicht ausschließlich an Bedarfskriterien, sondern mehr an patientenrelevanten Outcomes, 
auch in Relation zu den aufgewendeten Kosten, sowie dafür relevanten Rahmenbedingungen auf regionaler wie auf Bundesebene orientiert werden.

\section{Korrespondenzadresse}

\section{Dr. Antje Freytag}

Institut für Allgemeinmedizin, Universitätsklinikum Jena

Bachstr. 18, 07743 Jena, Deutschland antje.freytag@med.uni-jena.de

Funding. Open Access funding enabled and organized by Projekt DEAL.

\section{Einhaltung ethischer Richtlinien}

Interessenkonflikt. B. Ditscheid, M. Krause, T. Lehmann, K. Stichling, M. Jansky, F. Nauck, U. Wedding, W. Schneider, U. Marschall, W. Meißner und A. Freytag geben an, dass kein Interessenkonflikt besteht.

Ein positives Ethikvotum wurde von der Ethikkommission des Universitätsklinikums Jena erteilt (531710/17). Für diesen Beitrag wurden von den Autoren keine Studien an Menschen und Tieren durchgeführt.

Open Access. Dieser Artikel wird unter der Creative Commons Namensnennung 4.0 International Lizenz veröffentlicht, welche die Nutzung, Vervielfältigung, Bearbeitung, Verbreitung und Wiedergabe in jeglichem Medium und Format erlaubt, sofern Sie den/die ursprünglichen Autor(en) und die Quelle ordnungsgemäß nennen, einen Link zur Creative Commons Lizenz beifügen und angeben, ob Änderungen vorgenommen wurden.

Die in diesem Artikel enthaltenen Bilder und sonstiges Drittmaterial unterliegen ebenfalls der genannten Creative Commons Lizenz, sofern sich aus der Abbildungslegende nichts anderes ergibt. Sofern das betreffende Material nicht unter der genannten Creative Commons Lizenz steht und die betreffende Handlung nicht nach gesetzlichen Vorschriften erlaubt ist, ist für die oben aufgeführten Weiterverwendungen des Materials die Einwilligung des jeweiligen Rechteinhabers einzuholen.

Weitere Details zur Lizenz entnehmen Sie bitte der Lizenzinformation auf http://creativecommons.org/ licenses/by/4.0/deed.de.

\section{Literatur}

1. Gemeinsamer Bundesausschuss (2010) Richtlinie des Gemeinsamen Bundesausschusses zur Verordnung von spezialisierter ambulanter Palliativversorgung

2. Prütz F, Saß A-C (2017) Daten zur Palliativversorgung in Deutschland. Bundesgesundheitsblatt Gesundheitsforschung Gesundheitsschutz 60(1):26-36. https://doi.org/10.1007/s00103016-2483-8

3. Robert Koch Institut (Hrsg) (2015) Gesundheit in Deutschland. Gesundheitsberichterstattung des Bundes. RKI, DESTATIS, Berlin
4. Jope I, Wegleitner K, Heimerl K (2011) Expertise zur Ist-Stand-Analyse der Hospiz- und Palliativversorgung und Bedarfsanalyse der stationären Hospizund Palliativversorgung in Thüringen (Im Auftrag des Thüringer Hospiz- und Palliativverbandes (THPV), Meiningen, Jena)

5. Landesarbeitsgemeinschaft HOSPIZ Saarland e. V. (2013) Stellungnahme zum mittelfristigen Bedarf der Hospiz- und Palliativversorgung im Saarland

6. Hesse M, Ates G (2017) Hospiz- und Palliativversorgung in Rheinland-Pfalz. Gutachten im Auftrag des Ministeriums für Soziales, Arbeit, Gesundheit und Demografie des Landes Rheinland-Pfalz (MSAGD)

7. Weinhold I, Claus F, Karmann A, Kliemt R, Wende D, Plaul C (2018) Hospizstudie 2017. Standorte und demografische Rahmenbedingungen zur Hospizund Palliativversorgung im Freistaat Sachsen. Gutachten im Auftrag des Sächsischen Staatsministeriums für Soziales und Verbraucherschutz

8. van den Berg N, Rehner L, Hoffmann W (2018) Die Hospiz- und Palliativversorgung in MecklenburgVorpommern. Im Auftrag des Landtags Mecklenburg-Vorpommern (LT-Drs.Nr. 7/80), Greifswald

9. Meyer I, Schubert I (2020) Inanspruchnahme von SAPVund weiteren palliativen Leistungen, Charakteristika der Leistungsempfänger und Hinweise auf die Zielerreichung von SAPV in der Region Nordrhein. In: Krumm N, Rolke R (Hrsg) Evaluation der Wirksamkeit von SAPVin Nordrhein, S 27-134 (Bericht zur retrospektiven Sekundärdatenanalyse [AP1.1] im Projekt APVEL Ambulante Palliative Versorgung Evaluieren)

10. Radbruch L, Andersohn F, Walker J (2015) Palliativversorgung - Modul 3. Überversorgung kurativ - Unterversorgung palliativ? Analyse ausgewählter Behandlungen am Lebensende. Faktencheck Gesundheit

11. Freytag A, Krause M, Bauer A et al (2019) Study protocol for a multi-methods study: SAVOIR evaluation of specialized outpatient palliative care (SAPV) in Germany: outcomes, interactions, regional differences. BMC Palliat Care 18(1):12. https://doi.org/10.1186/s12904-019-0398-5

12. Stiel $S$, Krause $O$, Berndt CS, Ewertowski H, MüllerMundt G, Schneider N (2019) Caring for frail older patients in the last phase of life: Challenges for general practitioners in the integration of geriatric and palliative care \{Versorgung älterer gebrechlicher Patienten in der letzten Lebensphase: Herausforderungen für Hausärzte bei der Integration geriatrischer und palliativer Versorgungsansätze]. Z Gerontol Geriatr. https:// doi.org/10.1007/s00391-019-01668-3

13. Stiel S, Ewertowski H, Krause O, Schneider N (2019) Hausärztliche Versorgung von älteren gebrechlichen Patienten in der letzten Lebensphase zwischen geriatrischen und palliativmedizinischen Ansätzen

14. Murtagh FE, Bausewein C, Verne J, Groeneveld El, Kaloki YE, Higginson IJ (2014) How many people need palliative care? A study developing and comparing methods for population-based estimates. Palliat Med 28(1):49-58. https://doi. org/10.1177/0269216313489367

15. Scholten N, Günther AL, Pfaff H, Karbach U (2016) The size of the population potentially in need of palliative care in Germany-An estimation based on death registration data. BMC Palliat Care 15(1):29. https://doi.org/10.1186/s12904016-0099-2

16. Morin L, Aubry R, Frova L et al (2017) Estimating the need for palliative care at the population level: a cross-national study in 12 countries.
Palliat Med 31(6):526-536. https://doi.org/10. $1177 / 0269216316671280$

17. Melching H (2015) Palliativversorgung. Strukturen und regionale Unterschiede in der Hospiz- und Palliativversorgung. Faktencheck Gesundheit

18. GKV-Spitzenverband (2017) Bericht des GKVSpitzenverbandes zur Palliativversorgung

19. Jansky M, Lindena G, Nauck F (2011) Stand der spezialisierten ambulanten Palliativversorgung (SAPV) in Deutschland -Verträge und Erfahrungen [Specialized out-patient palliative care (SAPV) in Germany - Contracts and experiences]. Palliativmedizin 12(04):164-174. https://doi.org/10.1055/ s-0031-1276839

20. GKV-Spitzenverband Rahmenvereinbarung nach § 39a Abs. 1 Satz 4 SGB V über Art und Umfang sowie Qualität der stationären Hospizversorgung vom 13.03.1998, i.d. F. vom 31. März 2017

21. Deutsche Gesellschaft für Palliativmedizin Wegweiser Hospiz- und Palliativversorgung Deutschland (2017) Übersicht zum aktuellen Stand der Hospiz- und Palliativversorgung in Deutschland. https://www.dgpalliativmedizin. de/images/stories/pdf/161231_Wegweiser\%C3 $\%$ BCbersicht_Homepage_Erwachsene.pdf.Zugegriffen: 3. Juni 2020

22. Stichling K, Krause M, Ditscheid B et al (2020) Factors inflencing GPs' perception of specialised palliative homecare (SPHC) importance-results of a cross-sectional study. BMC Palliat Care 19:117. https://doi.org/10.1186/s12904-020-00603-3

23. Bayerisches Staatsministerium für Umwelt und Gesundheit, Bayerisches Staatsministerium für Arbeit, Sozialordnung, Familie und Frauen (2011) Begleitung und Versorgung Schwerstkranker und Sterbender sowie ihrer Angehörigen in Bayern. Rahmenkonzept zur Hospiz- und Palliativversorgung

24. Der Senat von Berlin (2011) 3. Hospiz- und Palliativkonzept für das Land Berlin. Fortschreibung des Hospizkonzeptes von 1998 (Hospizkonzept - Förderung der Hospizentwicklung im Land Berlin) (Fortschreibung des Hospizkonzeptes von 1998 (Hospizkonzept - Förderung der Hospizentwicklung im Land Berlin) und des im Jahr 2006 vorgelegten Berichts zur Fortschreibung des Hospizkonzeptes. Drucksache 16/4057)

25. Stichling K (2019) Hausärztliche Betreuung von Palliativpatienten innerhalb und außerhalb der SAPV - eine Querschnittserhebung im Rahmen des Verbundprojektes "SAVOIR - Evaluierung der SAPV-Richtlinie: Outcomes, Interaktionen, Regionale Unterschiede". Dissertation, FriedrichSchiller-Universität

26. Mühlensiepen $F$, von Peter $S$, Thoma $S$ et al (2019) Pilotprojekt zur Stärkung der ambulanten Palliativversorgung im Land Brandenburg - Analyse von Dokumentationsdaten der allgemeinen ambulanten Palliativversorgung [Pilot project: reinforcement of outpatient palliative care in the State of Brandenburg - An analysis of documentation data of primary palliative care]. Gesundheitswesen. https://doi.org/10.1055/a0905-2974

27. Kassenärztliche Bundesvereinigung (2019) Vertragsärzte und-psychotherapeuten je 100.000 Einwohner nach Fachgruppen und Regionen für die Jahre 2014 bis 2018 - Statistische Informationen aus dem Bundesarztregister der Kassenärztlichen Bundesvereinigung. Versorgungsatlas-Bericht, Bd. $19 / 09$

28. Simmenroth-Nayda A, Gágyor I (2008) Wem gehört die ambulante Palliativmedizin? ZAllg Med 


\section{Originalien und Übersichten}

84(6):236-238. https://doi.org/10.1055/s-20081080901

29. HAGE - Hessische Arbeitsgemeinschaft für Gesundheitsförderung e. V., Arbeitsbereich KASA, HPVH e. V., Fachverband SAPV (2018) Hospizarbeit und palliative Versorgung in Hessen. Praxishilfe für die Hospiz- und Palliativarbeit vor OrtBd. 3

30. Niedersächsisches Ministerium für Soziales, Frauen, Familie und Gesundheit (2006) Rahmenkonzept zur Weiterentwicklung der Palliativversorgung in Niedersachsen

31. Niedersächsisches Ministerium für Soziales, Frauen, Familie, Gesundheit und Integration (2013) Weiterentwicklung der Hospizarbeit und Palliativversorgung in Niedersachsen. (1. Fortschreibung des Rahmenkonzepts zur Weiterentwicklung der Palliativversorgung in Niedersachsen vom März 2006)

32. Ministerium für Gesundheit, Soziales, Frauen und Familie des Landes Nordrhein-Westfalen (2005) Rahmenprogramm zur flächendeckenden Umsetzung der ambulanten palliativmedizinischen und palliativpflegerischen Versorgung in NRW. Kooperatives integratives Versorgungskonzept

33. KV Nordrhein (2015) Vereinbarung über die palliativmedizinische Versorgung von unheilbar erkrankten Versicherten im häuslichen Umfeld (Ersatzkassen)

34. KV Nordrhein (2009) Vertrag über die Erbringung Spezialisierter ambulanter Palliativversorgung (SAPV) in Nordrhein (Ersatzkassen)

35. KV Westfalen-Lippe (2013) Vereinbarung zur Umsetzung der ambulanten palliativmedizinischen Versorgung von unheilbar erkrankten Patienten im häuslichen Umfeld

36. Lux EA, Hofmeister U, Bornemann R (2016) Ambulante Palliativversorgung in WestfalenLippe - Eine Follow-up-Erhebung fünf Jahre nach Einführung einer strukturierten Versorgung im häuslichen Umfeld [Palliative approach in outpatient care in Westphalia-Lippe-Follow-up study five years after inception of a structured home-based palliative care system]. Dtsch Med Wochenschr 141(8):e67-e72. https://doi.org/10. 1055/s-0041-106677

37. Hofmeister U (2015) Wichtige Aspekte einer patientenorientierten Palliativversorgung in Westfalen-Lippe. Faktencheck Gesundheit 